\title{
WATER, ENERGY AND LIFE: FRESH VIEWS FROM THE WATER'S EDGE
}

\author{
G.H. POLLACK \\ Department of Bioengineering, University of Washington, Seattle, Washington, USA.
}

\begin{abstract}
Recent observations have shown an unexpected feature of water adjacent to hydrophilic surfaces: the presence of wide interfacial zone that excludes solutes. The exclusion zone is charged, while the water beyond is oppositely charged, yielding a battery-like feature. The battery is powered by absorbed radiant energy. Implications of this energetic feature are discussed. It appears that the presence of this 'exclusion zone' may play an important role in the behavior of aqueous systems.

Key words: exclusion zone, hydrophilic surfaces, interfacial water, light, radiant energy.
\end{abstract}

The impact of surfaces on the contiguous aqueous phase is generally thought to extend no more than a few water-molecule layers. We find, however, that this prevailing view does not hold. Solutes are profoundly excluded from the vicinity of various hydrophilic surfaces to distances typically several hundred micrometers. Numerous controls have shown that such exclusion is not the trivial result of some unsuspected artifact [1], and by now such long-range exclusion has been confirmed in a dozen different laboratories worldwide. We term these apparently solute-free regions 'exclusion zones'.

Exclusion zones are observed next to many hydrophilic surfaces, but not next to hydrophobic surfaces. The classes of hydrophilic surfaces tested include hydrogels, hydrophilic polymers, monolayers, ion-exchange beads and biological tissues. The exclusion zones next to these surfaces exclude a diverse array of solutes of various size, type and polarity. Hence, the exclusion phenomenon is quite general $[2,3]$. An example of an exclusion zone next to a hydrophilic gel surface is shown in Fig. 1.

Six methods have so far been applied to test whether the physical properties of exclusion-zone water differ from those of bulk water. They include the following: NMR, infrared and birefringence imaging, and measurements of electrical potential, viscosity and UV-VIS absorption spectra. All approaches show that the interfacial zone differs physically from the bulk zone, and that it is a distinct, less mobile, more ordered phase of water that can coexist with the contiguous bulk phase [2]. Hence, the exclusion zone appears to behave like a liquid crystal, i.e. a phase of water in which constituent molecules are more-or-less lined up.

Of the results mentioned above, a particularly significant one is charge separation. Although the overall net charge of water in the system is zero, water in the exclusion zone is negatively charged, while the bulk-water region beyond contains positive charges. The potential difference is of the order of 100-200 mV, depending on the type of nucleating surface. Electrodes inserted into the respective zones and connected through a resistor show ample, persisting, current flow.

Charge separation in water may seem counterintuitive, but it is common in other realms. In cloud water for example, evidence of charge separation lies in the consequent lightning discharges, $80 \%$ of which go from cloud to cloud. In the laboratory, the famous Kelvin water-dropper experiment (modern incarnations easily found on the web) shows visible electric discharge between juxtaposed bodies of pure water, again indicating water-based charge separation. Charge separation has also been demonstrated in our laboratory. In a chamber filled with pure water, electrodes placed at either end of the bath

(C) 2010 WIT Press, www.witpress.com

ISSN: 1755-7437 (paper format), ISSN: 1755-7445 (online), http://journals.witpress.com DOI: 10.2495/DNE-V5-N1-27-29 


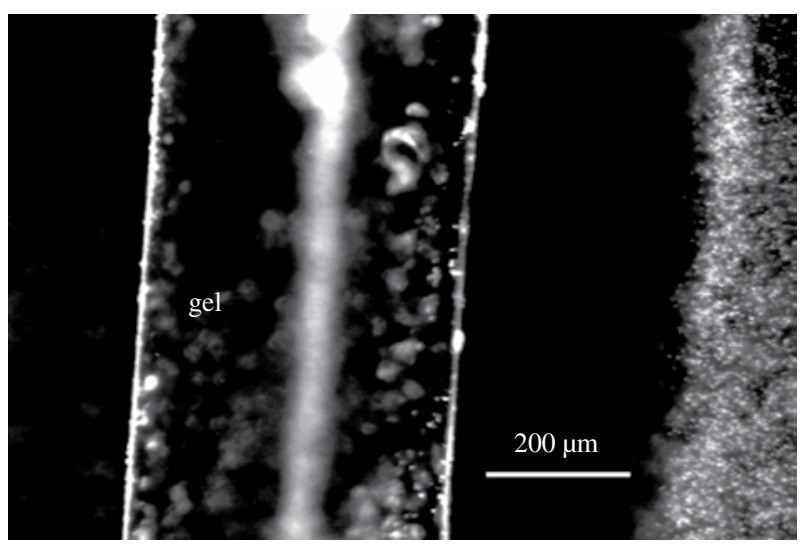

Figure 1: Solute exclusion in the vicinity of polyacrylic-acid gel. The gel was placed on a coverslip, superfused with a suspension of $1-\mu \mathrm{m}$ carboxylate-coated microspheres and observed in an inverted microscope. Image was obtained $20 \mathrm{~min}$ after superfusion. (Fuzzy vertical line within gel is optical artifact.) Microspheres move away from gel, leaving regions (black) on either side of gel that are devoid of them. Microspheres seen on right edge of figure.

and driven to pass current create ample charge buildup in the water: the half nearest the anode remains positive, while the half nearest the cathode remains negative, even well after the driver has been disconnected [4]. Such stored water charge turns out to be largely recoverable [5]. These three examples offer ample precedent for charge separation in water - as we observe near hydrophilic surfaces.

For building the charged exclusion zone, the energy apparently comes from light. We found that incident radiant energy, including UV, visible and near-infrared wavelengths, induces exclusionzone growth. IR is especially effective: at wavelength $3.1 \mu \mathrm{m}$, a 10-minute exposure to incident radiation - weak enough to elevate water temperature by no more than $1{ }^{\circ} \mathrm{C}$ - causes the exclusionzone width to increase up to four times [6]. This is an impressive increase. Apparently, photons induce some change in water that predisposes constituent $\mathrm{OH}^{-}$and $\mathrm{H}^{+}$moieties to reorganize, the former building the negatively charged exclusion zone and leaving the bulk zone positively charged with hydronium ions. We have gone so far as to exploit this light-induced charge separation for obtaining clean electrical energy (patent pending).

Photons from sunlight, then, may exert an unexpected effect that goes well beyond mere heating. Solar energy incident on water evidently separates charge. Such light-induced near-surface water splitting is what happens in reaction centers as the first step of photosynthesis. Hence, the phenomenon identified here may be something akin to an initial step in photosynthesis - a kind of 'generic' first step that occurs next to many hydrophilic surfaces rather than only next to those specific to green plants or bacteria. This could represent a potentially significant energetic pathway that may be highly relevant for animal biology (and medicine).

To those unfamiliar with surfaces and water, the claims above may seem extraordinary. Given the intensity of scientific activity, how could others possibly have failed to come upon so simple a finding? In fact, many have. An early review [7] cited more than a hundred references showing that for many liquids including water, the near-surface zones differ in their physical chemical properties from regions farther away. These zones could extend as far as 'hundreds of microns' from the surface. Unfortunately, as interest shifted increasingly away from water during the latter part of the last century, this body of evidence faded from the forefront and was eventually forgotten. 
On the other hand, a later observation by Green and Otori [8] produced results almost identical to our own (an embarrassment when their paper was uncovered). Exploring the basis of the so-called unstirred layer - the extremely slow-diffusion region immediately surrounding many biological tissues - these investigators exposed natural and artificial tissues to microsphere suspensions. The near-surface regions profoundly excluded the microspheres. The zones of exclusion were typically several hundred micrometers wide, and could not be eliminated by vigorous stirring. The authors concluded that water in the near-tissue zone was physically different from water in the bulk, and that such difference could explain the existence of the long known and extensively studied unstirred layer (for review, c.f. [9]). Unfortunately, the authors apparently never went on to explore the basis for the observed physicochemical difference.

The point is that the claims above about long-range water ordering are not all original. Many investigators, including Nobel laureate Albert Szent-Gyorgyi, who concluded following many studies that 'life is water dancing to the tune of solids', have provided ample evidence that interfacial water is profoundly different from water in the bulk. It is merely a matter of exploring the detailed nature of this water, and its impact on nature.

For additional details of the evidence mentioned here, the reader is referred to a recent public lecture accessible on YouTube, Google Video or on http://uwtv.org/programs/displayevent. aspx?rID=22222. This hour-long lecture concisely summarizes the basic and most recent evidence, and limns these findings' broad scientific and technological implications. The potential significance of these new findings for biology, medicine and many other aspects of nature appear to be quite profound.

In conclusion, this short essay merely scratches the surface of hydrophilic interfaces. The finding of substantial interfacial exclusion zones that separate charge - and especially that the separation is driven by radiant energy - opens many doors to understanding. One hope is that this fundamental finding may help answer many lingering questions and bring new insights.

\section{REFERENCES}

[1] Zheng, J.M. \& Pollack, G.H., Long range forces extending from polymer surfaces. Physical Review E, 68, pp. 031408, 2003. doi:10.1103/PhysRevE.68.031408

[2] Zheng, J.M., Chin, W.C, Khijniak, E., Khijniak, E. Jr. \& Pollack, G.H., Surfaces and Interfacial Water. Evidence that hydrophilic surfaces have long-range impact. Advances in Colloid and Interface Science, 127, pp.19-27, 2006. doi:10.1016/j.cis.2006.07.002

[3] Zheng, J.M. \& Pollack, G.H., Solute exclusion and potential distribution near hydrophilic surfaces. Water and the Cell, eds G.H. Pollack, I.L. Cameron \& D.N. Wheatley, Springer, pp. 165-174, 2006.

[4] Klimov, A. \& Pollack, G.H., Visualization of charge-carrier propagation in water. Langmuir 23(23), pp. 11890-11895, 2007. doi:10.1021/la701742v

[5] Ovchinnikova, K \& Pollack, G.H., Can water store charge? Langmuir, 25, pp, 542-547, 2009.

[6] Chai, B., Yoo, H. \& Pollack, G.H., Effect of radiant energy on near-surface water. J. Phys. Chem. B, 113, pp. 13953-13958, 2009. doi:10.1021/jp908163w

[7] Henniker, J.C., The depth of the surface zone of a liquid. Reviews of Modern Physics, 21(2), pp, 322-341, 1949.

[8] Green, K. \& Otori, T., Direct measurement of membrane unstirred layers. The Journal of Physiology, 207, pp.93-102, 1970.

[9] Pollack, G.H. \& Clegg, J., Unsuspected linkage between unstirred layers, exclusion zones, and water. Phase Transitions in Cell Biology, eds Pollack, G.H. \& Chin, W.C., Springer, pp. 143-152, 2008. 